\title{
Low-temperature sintering and hot rolling of hard powder magnetic Fe-Cr-Co alloy
}

\author{
A. S. Ustyukhin ${ }^{\dagger}$ A. B. Ankudinov, V. A. Zelenskiy, I. M. Milyaev, M. I. Alymov \\ †fcbneo@yandex.ru
}

A. A. Baikov Institute of Metallurgy and Materials Science RAS. 49 Leninskiy prospekt, 119334, Moscow, Russia

In the present work, a method for processing of a technically usable Fe-26\%Cr-16\%Co-2\%Mo-2\%W hard magnetic alloy from powder raw materials is proposed. The distinctive feature of the method is the lowered sintering temperature of powder compacts $\left(1100-1200^{\circ} \mathrm{C}\right)$. Sintered blanks are subjected to a hot rolling process at $1150^{\circ} \mathrm{C}$. The magnetic material produced by the proposed method has a relative density of about 97 to $98 \%$. Magnetic properties of the alloy sintered at $1200^{\circ} \mathrm{C}$ $\left(\mathrm{B}_{\mathrm{r}}=1,2 \mathrm{~T}, \mathrm{H}_{\mathrm{cb}}=51 \mathrm{kA} / \mathrm{m},(\mathrm{BH})_{\max }=30 \mathrm{~kJ} / \mathrm{m}^{3}\right)$ are on the same level as for alloys obtained by powder metallurgy methods with sintering temperatures higher than $1300^{\circ} \mathrm{C}$ and their cast analogs. At the same time, low magnetic properties were obtained on the alloy sintered at $1100^{\circ} \mathrm{C}$ with the following hot rolling. Using EDX analysis and X-ray phase analysis it has been established that this is due to the presence of a significant fraction of non-magnetic $\gamma$-phase in the samples. Apparently, under these sintering conditions the redistribution of alloy components does not succeed. Tensile strength tests of the hot-rolled alloy after heat treatment showed a significant effect of sintering temperature on the samples strength. The tensile strength was higher for the samples sintered at $1100^{\circ} \mathrm{C}(700-930 \mathrm{MPa})$ as compared to the samples sintered at $1200^{\circ} \mathrm{C}(520-590 \mathrm{MPa})$. Microstructural studies of the samples showed that alloy sintered at $1200^{\circ} \mathrm{C}$ had a more coarse-grained structure. Apparently, this is a cause of the tensile strength reduction.

Keywords: powder metallurgy, hard magnetic Fe-Cr-Co alloys, hot rolling, magnetic hysteresis properties, mechanical properties.

\section{Низкотемпературное спекание и горячая прокатка магнитотвердого порошкового сплава системы Fe-Cr-Co}

\author{
Устюхин А. С. ${ }^{\dagger}$ Анкудинов А. Б., Зеленский В. А., Миляев И. М., Алымов М.И. \\ †fcbneo@yandex.ru
}

Институт металлургии и материаловедения им. А.А. Байкова РАН, Ленинский проспект 49, 119334, Москва, Россия

В работе предлагается способ получения технически пригодного магнитотвердого материала состава $\mathrm{Fe}-26 \% \mathrm{Cr}-$ 16\%Сo-2\%Мо-2\%W из порошкового сырья. Отличительной особенностью метода является пониженная температура спекания порошковых прессовок $\left(1100-1200^{\circ} \mathrm{C}\right)$. Спеченные заготовки подвергались процедуре горячей прокатки при $1150^{\circ} \mathrm{C}$. Изготовленный предложенным способом магнитный материал имел относительную плотность порядка

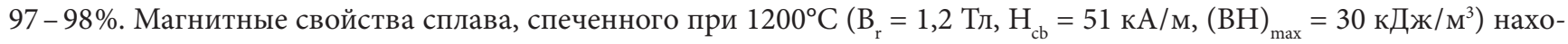
дятся на уровне свойств сплавов, полученных методами порошковой металлургии при температуре спекания выше $1300^{\circ} \mathrm{C}$ и их литых аналогов. В то же время, на сплаве, спеченном при $1100{ }^{\circ} \mathrm{C}$ с последующей горячей прокаткой были получены низкие магнитные свойства. С помощью методов МРСА и рентгенофазового анализа установлено, что это связано с наличием значительной доли немагнитной $\gamma$-фазы в образцах. По всей видимости, при этих условиях спекания не успевает произойти перераспределение компонентов сплава. Испытания на прочность при растяжении прокатанного сплава после термообработки показали существенное влияние температуры спекания на прочность образцов. При этом прочность на растяжение оказалась выше у образцов, спеченных при $1100^{\circ} \mathrm{C}(700-930 \mathrm{MПа})$ по сравнению с образцами, спеченными при $1200^{\circ} \mathrm{C}$ (520-590 МПа). Исследование микроструктуры прокатанных образцов показало, что сплав, спеченный при $1200^{\circ} \mathrm{C}$, имеет более крупнозернистую структуру. По всей видимости, это и приводит к снижению прочности.

Ключевые слова: порошковая металлургия, магнитотвердые сплавы Fe-Cr-Co, горячая прокатка, магнитные гистерезисные свойства, механические свойства. 


\section{1. Введение}

Производство магнитотвердых материалов системы $\mathrm{Fe}-\mathrm{Cr}-\mathrm{Co}$ для постоянных магнитов порошковым методом имеет ряд преимуществ по сравнению с технологией литья: возможность прецизионного регулирования химического состава сплавов, повышенный коэффициент использования металла при производстве, повышенная производительность, высокие магнитные свойства. Магнитные свойства сплавов системы Fe-Cr-Co удачно сочетаются с высокой коррозионной стойкостью, прочностью при достаточной пластичности [1-7]. Указанные сплавы характеризуются высокими гистерезисными свойствами: остаточной индукцией $\mathrm{B}_{\mathrm{r}}=1,15-1,30$ Тл, коэрцитивной силой $\mathrm{H}_{\text {св }}=36-52$ кА/м, максимальным энергетическим произведением (BН $)_{\max }=30-36$ кДж/ $\mathrm{M}^{3}$, высокой температурно-временной стабильностью - рабочие температуры до $500-550^{\circ} \mathrm{C}$. В отличие от других магнитотвердых материалов, сплавы системы Fe-Cr-Co в высококоэрцитивном состоянии обладают высокой механической прочностью на уровне 600-900 МПа при пластичности $2-4 \%$. Эти свойства делают их незаменимыми для изготовления постоянных магнитов, работающих в условиях высоких статических и динамических нагрузок и агрессивных сред. Объемы производства магнитов из сплавов системы Fe-Cr-Co неуклонно растут. Постоянно идет и совершенствование технологии производства.

При традиционном производстве магнитных сплавов системы Fe-Cr-Co методами порошковой металлургии применяют высокую температуру спекания прессовок (около $1400^{\circ} \mathrm{C}$ ) [8-10], что требует больших энергозатрат. При высокой температуре с поверхности прессовок происходит частичное испарение компонент исходной шихты, меняется общий химический состав, появляется химическая неоднородность материала по объему. Наибольшему испарению подвержен хром [11]. Этот процесс может приводить к ухудшению свойств, особенно малогабаритных изделий, когда в приповерхностных слоях находится основной объем материала магнита.

В данной работе предлагается уменьшить температуру спекания порошковых прессовок и тем самым снизить отрицательное воздействие испарения. Процесс проводили при температурах 1100 и $1200^{\circ} \mathrm{C}$. Недостаточная плотность материала, обусловленная понижением температуры спекания, устраняется последующей прокаткой, в ходе которой уменьшается пористость заготовок при обжатии. Приводятся результаты исследований характеристик полученных таким способом материалов.

\section{2. Методика эксперимента}

Bсе эксперименты были выполнены на магнитотвердом порошковом материале состава $\mathrm{Fe}-26 \% \mathrm{Cr}-16 \% \mathrm{Co}-$ $2 \% \mathrm{Mo}-2 \% \mathrm{~W}$. Для изготовления образцов использовали порошки: кабонильное железо марки ВС; хром ПХС-1, кобальт ПК-1У; молибдена и вольфрама с частицами $<20$ мкм.

Изготовление прямоугольных прессовок производили в разъёмной матрице с размерами $82,3 * 16,2$ *9,8 мм.
Прессование осуществляли на прессе П-250.

Спекание прессовок проводили в вакуумной шахтной печи СШВ-1,25/24-И1. Вакуум был не хуже $10^{-2}$ Па. Образцы выдерживали 150 минут при температуре спекания.

Плотность прессовок и спеченных образцов определяли измерением массы и геометрических размеров. Значения плотности прокатанных образцов определяли методом гидростатического взвешивания.

Измерения магнитных гистерезисных свойств производили на гистерезисграфе Permagraph L. Погрешность измерений коэрцитивной силы $\mathrm{H}_{\text {c }}$ и остаточной индукции $\mathrm{B}_{\mathrm{r}}$ составляла $3 \%$, магнитного произведения $(\mathrm{BH})_{\max }-6 \%$.

Микрорентгеноспектральный анализ (MPCA) был выполнен на рентгеновском микроанализаторе “Superprobe JCXA-733” фирмы “JEOL” с приставкой EDA INCA-300 Oxford Instruments (EDA-энергодисперсионный анализ).

Механические испытания проводили на установке Instron 3382.

Для исследования микроструктуры применялась оптическая системы анализа изображений на базе микроскопа OLYMPUS PME-3. Для выявления микроструктуры использовали царскую водку и смесь концентрированной соляной кислоты + хлорное железо + этиловый спирт в соотношении $2: 3: 10$.

\section{3. Результаты и обсуждение}

В наших ранних работах $[12,13]$ было показано, что применение высокой температуры спекания при получении порошковых магнитотвердых сплавов системы $\mathrm{Fe}-\mathrm{Cr}-\mathrm{Co}$ вызывает частичное испарение хрома из приповерхностных слоев (рис. 1).

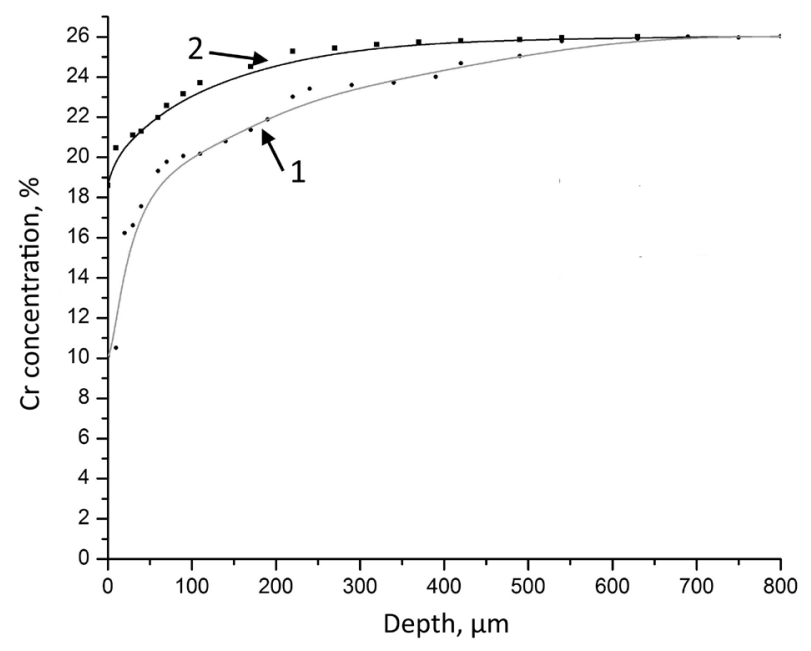

Рис. 1. Пространственные распределения концентрации хрома, полученные с помощью метода МРСА после спекания сплава Fe-26\%Cr-16\%Co-2\%Mo-2\%W при температурах: (1) $-1420^{\circ} \mathrm{C},(2)-1330^{\circ} \mathrm{C}$

Fig. 1. Chromium concentration profiles determined via EDX analysis for samples of $\mathrm{Fe}-26 \% \mathrm{Cr}-16 \% \mathrm{Co}-2 \% \mathrm{Mo}-2 \% \mathrm{~W}$ alloy sintered at (1) $1420^{\circ} \mathrm{C}$ and (2) $1330^{\circ} \mathrm{C}$. 
Испарение хрома в свою очередь приводит к возникновению химической неоднородности, что отрицательно сказывается на магнитных свойствах сплава. $\mathrm{H}_{\text {c }}$ и $\mathrm{B}_{\mathrm{r}}$ снижаются на $5-10 \%$, а $(\mathrm{BH})_{\max }-$ на $10-15 \%$. Таким образом, спекание в вакууме при температуре порядка $1400^{\circ} \mathrm{C}$ зачастую не обеспечивает необходимого уровня магнитных свойств особенно малогабаритных изделий, несмотря на высокую плотность материала.

Для уменьшения воздействия испарения в настоящих исследованиях температура спекания порошковых прессовок была снижена. Процесс проводили при температурах 1100 и $1200^{\circ} \mathrm{C}$. Недостающую плотность материала, обусловленную пониженной температурой спекания, обеспечили последующей прокаткой, в ходе которой пористость заготовок при обжатии уменьшалась.

Прямоугольные прессовки получали при давлении прессования 250 МПа. Они имели относительную плотность около $67 \%$. После спекания при температуре $1100^{\circ} \mathrm{C}$ плотность имела в среднем значение 86\%, при температуре спекания $1200^{\circ} \mathrm{C}$ около $90 \%$ (см. табл. 1).

Далее для увеличения плотности образцы подвергали горячей продольной прокатке с семикратным обжатием в валках прокатного стана при температуре $1150^{\circ} \mathrm{C}$. Каждое последующее обжатие проводилось при уменьшении величины зазора между валками. Коэффициент вытяжки (отношение поперечных сечений до и после прокатки) составил 1,8-1,9. В результате плотность образцов была доведена до $97-98 \%$, что сопоставимо со сплавами такого же состава, спеченными при температуре выше $1300^{\circ} \mathrm{C}$. Следует отметить, что плотность прокатанных образцов оказалась независимой от температуры спекания прессовок.

Термообработку исследуемого сплава проводили после закалки образцов от $1250^{\circ} \mathrm{C}$ в воде. Скорость охлаждения в магнитном поле в температурном интервале $640-600^{\circ} \mathrm{C}$, т.е. в температурном интервале формирования высококоэрцитивного состояния, составила $40^{\circ} \mathrm{C} /$. Старение образцов после проведения ТМО проводили путём охлаждения в температурном интервале $600-480^{\circ} \mathrm{C}$ без магнитного поля со скоростью $8^{\circ} \mathrm{C} / ч$. Всю термообработку, включая термомагнитную, проводили в лабораторной установке с панцирным электромагнитом, обеспечивавшем напряжённость магнитного поля $\mathrm{H}=320 \mathrm{\kappa A} / \mathrm{M}$.
Результаты измерения магнитных гистерезисных свойств исследуемого сплава сведены в табл. 1. Для сравнения в ней также представлены данные по свойствам сплава непосредственно после спекания при вышеуказанных температурах и при тех же условиях ТМО.

Как видно из табл. 1, магнитные свойства всех образцов, спеченных при $1100^{\circ} \mathrm{C}$ имеют невысокие значения независимо от того применялась прокатка или нет. Следует отметить, что прокатка этих образцов только ухудшила магнитные характеристики, в особенности остаточную индукцию $\mathrm{B}_{\mathrm{r}}$ хотя плотность материала возрастает существенно. Магнитные свойства образцов, спеченных при $1200^{\circ} \mathrm{C}$, имеют приемлемые для технического применения магнитные характеристики. После прокатки заметно увеличились значения остаточной индукции $\mathrm{B}_{\mathrm{r}}$ при сохранении такого же уровня коэрцитивной силы $\mathrm{H}_{\text {св }}$. Было сделано предположение, что такая разница в магнитных свойствах при одинаковой плотности объясняется повышенной долей оксидных включений, которые образовались в процессе горячей прокатки на воздухе.

На рис. 2 представлены фотографии непротравленных шлифов прокатанных образцов, спеченных при разных температурах. Образцы были дополнительно закалены на однофазный $\alpha$-твёрдый раствор.

На светлом фоне заметны темные включения. Светлая матрица в образцах, спеченных при $1200^{\circ} \mathrm{C}$, является однородной (рис. 2b). Матрица образцов, спеченных при $1100^{\circ} \mathrm{C}$ (рис. 2a), состоит из участков серого цвета и более светлых. Результаты измерения содержания компонентов на различных участках поверхности шлифов представлены в табл. 2 .

Анализ показал, что темные включения являются местами скопления оксидных фаз. Количество включений и содержание кислорода в них практически одинаково для образцов, спеченных при разных температурах. Это указывает на то, что окисление в процессе горячей прокатки не является причиной резкого снижения магнитных свойств сплава, спеченного при $1100^{\circ} \mathrm{C}$. В образцах, спеченных при $1200^{\circ} \mathrm{C}$, наблюдается равномерное распределение компонентов в светлой матрице. Для образцов, спеченных при $1100^{\circ} \mathrm{C}$, заметна разница между серыми (фаза с повышенным содержанием Cr) и светлыми (фаза с повышенным содержанием Со) участками.

Табл. 1. Плотность и магнитные свойства спеченных и прокатанных образцов.

Table 1. Density and magnetic properties of sintered and rolled specimens.

\begin{tabular}{|c|c|c|c|c|c|}
\hline $\begin{array}{c}\text { Температура спекания, }{ }^{\circ} \mathrm{C} \\
\text { Sintering temperature }{ }^{\circ} \mathrm{C}\end{array}$ & $\begin{array}{l}\text { Прокатка } \\
\text { Hot rolling }\end{array}$ & $\begin{array}{l}\text { Плотность, \% } \\
\text { Density, \% }\end{array}$ & $\begin{array}{l}\mathrm{B}_{\mathrm{r}}, \text { Тл } \\
\mathrm{B}_{\mathrm{r}}, \mathrm{T}\end{array}$ & $\begin{array}{l}\mathrm{H}_{\mathrm{cB}}, \mathrm{KA} / \mathrm{M} \\
\mathrm{H}_{\mathrm{cB}}, \mathrm{kA} / \mathrm{m}\end{array}$ & 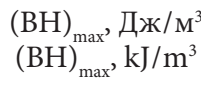 \\
\hline \multirow{2}{*}{1100} & $\begin{array}{c}\text { без прокатки } \\
\text { without rolling }\end{array}$ & $85-87$ & $0,79-0,83$ & $40-44$ & $11-14$ \\
\hline & $\begin{array}{l}\text { c прокаткой } \\
\text { with rolling }\end{array}$ & $97-98$ & $0,47-0,58$ & $40-41$ & $8,8-10,8$ \\
\hline \multirow{2}{*}{1200} & $\begin{array}{c}\text { без прокатки } \\
\text { without rolling }\end{array}$ & $89-91$ & $1,04-1,06$ & $51-53,5$ & $27,6-28,7$ \\
\hline & $\begin{array}{l}\text { с прокаткой } \\
\text { with rolling }\end{array}$ & $97-98$ & $1,19-1,21$ & $51-52$ & $29,6-31,3$ \\
\hline
\end{tabular}




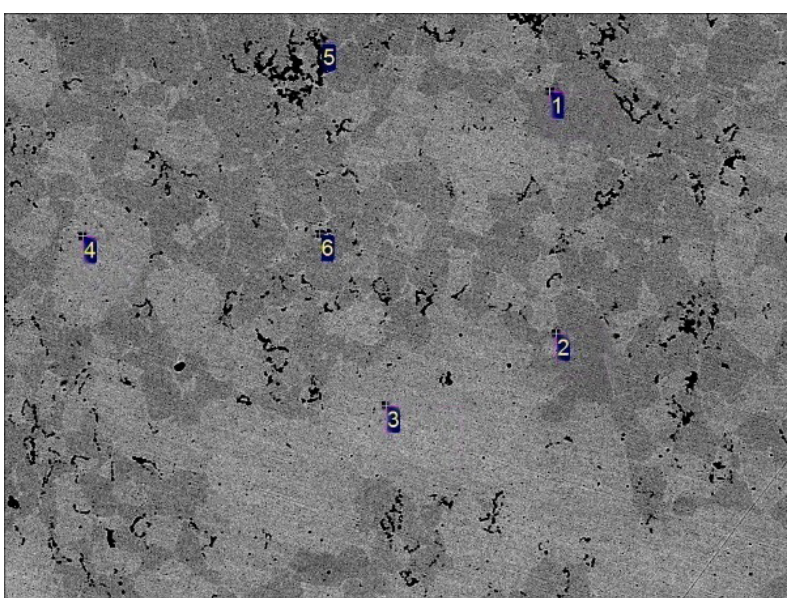

a

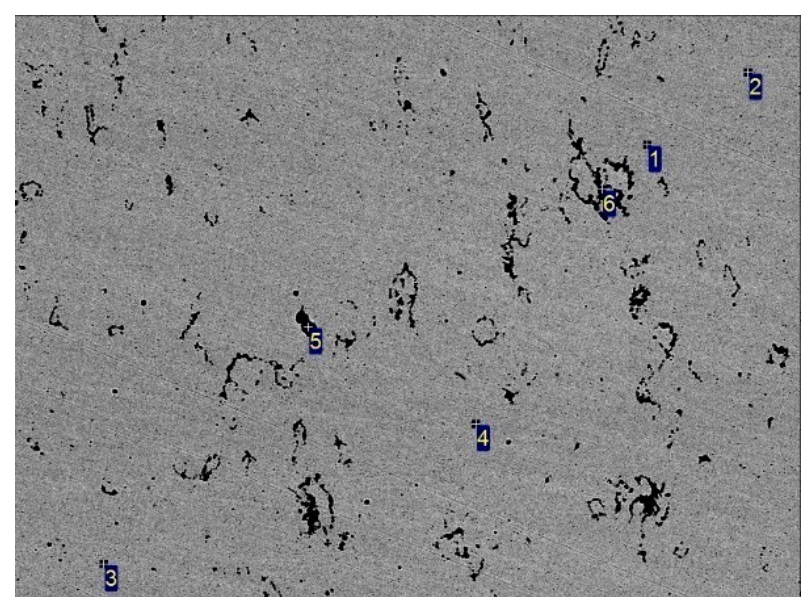

b

Рис. 2. Микрофотографии поверхности шлифов прокатанных образцов: температура спекания $1100^{\circ} \mathrm{C}(\mathrm{a})$ и $1200^{\circ} \mathrm{C}(\mathrm{b})$.

Fig. 2. Electron images of microsection surface of hot-rolled specimens sintered at (a) $1100^{\circ} \mathrm{C}$ and (b) $1200^{\circ} \mathrm{C}$.

табл. 2. Результаты измерения содержания компонентов методом МРCA в магнитном сплаве $\mathrm{Fe}-26 \% \mathrm{Cr}-16 \% \mathrm{Co}-2 \% \mathrm{Mo}-2 \% \mathrm{~W}$ (усредненные значения).

Table 2. The results of components content measurement via EDX analysis in Fe-26\%Cr-16\%Co-2\%Mo-2\%W magnetic alloy (average values).

\begin{tabular}{|c|c|c|c|c|c|c|c|c|}
\hline $\begin{array}{c}\text { Tемпература спекания, }{ }^{\circ} \mathrm{C} \\
\text { Sintering temperature, }{ }^{\circ} \mathrm{C}\end{array}$ & $\begin{array}{c}\text { Участок } \\
\text { Region }\end{array}$ & $\mathrm{O}, \%$ & $\mathrm{Cr}, \%$ & $\mathrm{Fe}, \%$ & $\mathrm{Co}, \%$ & $\mathrm{Mo}, \%$ & $\mathrm{~W}, \%$ & Total, \% \\
\hline \multirow{3}{*}{1100} & $\begin{array}{c}\text { серый } \\
\text { grey }\end{array}$ & 0.8 & 27.8 & 53.1 & 14.0 & 2.0 & 2.3 & 100.0 \\
\cline { 2 - 10 } & $\begin{array}{c}\text { светлый } \\
\text { light }\end{array}$ & 1.2 & 23.1 & 55.4 & 16.6 & 2.0 & 1.7 & 100.0 \\
\cline { 2 - 10 } & $\begin{array}{c}\text { темные включения } \\
\text { dark inclusions }\end{array}$ & 26.5 & 36.5 & 27.4 & 7.9 & 0.9 & 0.8 & 100.0 \\
\hline \multirow{2}{*}{1200} & $\begin{array}{c}\text { светлый } \\
\text { light }\end{array}$ & 1.3 & 25.8 & 54.5 & 14.6 & 1.9 & 1.9 & 100.0 \\
\hline & $\begin{array}{c}\text { темные включения } \\
\text { dark inclusions }\end{array}$ & 24.5 & 43.2 & 23.4 & 6.6 & 1.1 & 1.2 & 100.0 \\
\hline
\end{tabular}

Был проведен рентгенофазовый анализ образцов, который показал наличие немагнитной $\gamma$-фазы в прокатанных образцах, спеченных при $1100^{\circ} \mathrm{C}$ в течение 150 мин (рис. 3).

По всей видимости при этих условиях не успевает произойти перераспределение компонентов за счет диффузии, и даже после закалки на однофазный $\alpha$-твёрдый раствор в сплаве сохраняется значительная доля немагнитной $\gamma$-фазы. Нежелательное наличие $\gamma$-фазы влияет на дальнейшую термомагнитную обработку и приводит к значительному снижению магнитных гистерезисных свойств сплава.

Испытания на прочность при растяжении на прокатанных образцах проводили после полного цикла термообработки (включая ТМО и отпуск). Результаты измерений показали существенное влияние температуры спекания на прочность образцов. Прочность на растяжение оказалась выше у образцов, спеченных при $1100^{\circ} \mathrm{C}$ (700 - $930 \mathrm{MПа)} \mathrm{по} \mathrm{сравнению} \mathrm{с} \mathrm{образцами,} \mathrm{спеченными}$ при $1200^{\circ} \mathrm{C}$ (520-590 МПа). Пластичность образцов,

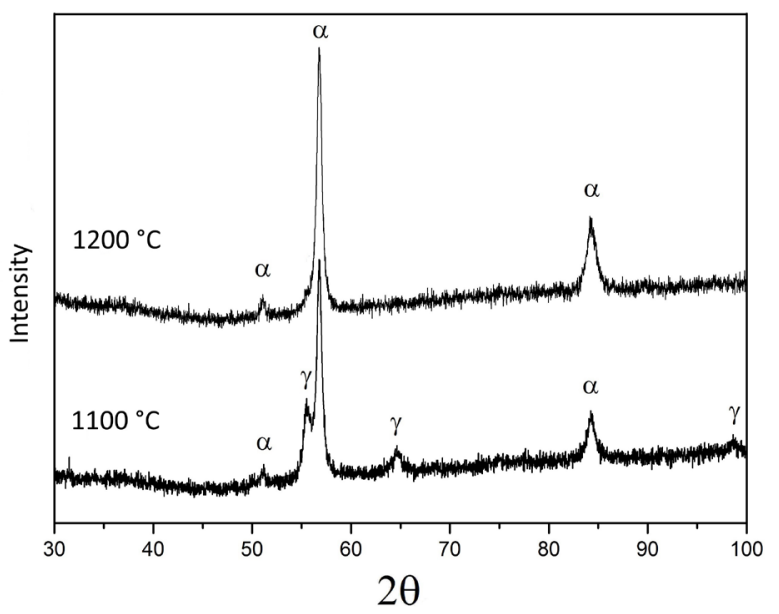

Рис. 3. Рентгенофазовый анализ прокатанных образцов сплава Fe-26\%Cr-16\%Co-2\%Mo-2\%W, спеченных по разным режимам.

Fig. 3. X-ray phase analysis of Fe-26\%Cr-16\%Co- $2 \% \mathrm{Mo}-2 \% \mathrm{~W}$ hotrolled specimens sintered at different conditions. 
спеченных при разных температурах, оказалась почти одинаковой и имела величину порядка $3 \%$.

Причина понижения прочности прокатанных образцов, спеченных при $1200^{\circ} \mathrm{C}$, по-видимому, объясняется структурой материала, в которой при этой температуре наблюдается рост зерна. На микрофотографии сплава, спеченного при $1100^{\circ} \mathrm{C}$ (рис. 4a), можно наблюдать как зерна размером порядка 50-100 мкм, так и более крупные. Микроструктура сплава, спеченного при $1200^{\circ} \mathrm{C}$ (рис. $4 \mathrm{~b}$ ), в основном состоит из зерен размером не менее 300-500 мкм. Вероятно, этим фактом и объясняется меньшая прочность на растяжение, прокатанного сплава, спеченного при $1200^{\circ} \mathrm{C}$.

\section{4. Выводы}

Показано, что плотный технически пригодный магнитотвердый порошковый материал состава $\mathrm{Fe}-26 \% \mathrm{Cr}$ $16 \%$ Со- $2 \%$ Мо- $2 \% \mathrm{~W}$ возможно получать при пониженной температуре спекания $\left(1200^{\circ} \mathrm{C}\right)$, если после спекания применять горячую прокатку. Плотность материала имеет величину $97-98 \%$, что сопоставимо со сплавами такого же состава, спеченными при температурах около $1400^{\circ} \mathrm{C}$.

На спеченном сплаве с последующей горячей прокаткой, получены следующие магнитные гистерезисные характеристики: $\mathrm{B}_{\mathrm{r}}=1,2 \mathrm{Tл}, \mathrm{H}_{\mathrm{cb}}=51 \mathrm{\kappa} A / \mathrm{m}$, $(\mathrm{BH})_{\max }=30 \mathrm{\kappa Дж} / \mathrm{M}^{3}$. Это соответствует государственным стандартам для литых аналогов.

Применение температуры спекания $1100^{\circ} \mathrm{C}$ ухудшает магнитные гистерезисные свойства прокатанного материала. Материал оказывается техническим не пригодным из-за наличия большой доли немагнитной $\gamma$-фазы в сплаве.

Предел прочности на растяжение прокатанного материала, спеченного при $1200^{\circ} \mathrm{C}$, имеет достаточную для технического применения величину ( 550 МПа). Материал, спеченный при $1100^{\circ} \mathrm{C}$, имеет повышенную прочность. Пластичность составляет порядка $3 \%$ и не зависит от температуры спекания.

Благодарность/Acknowledgements. Работа была выполнена при поддержке гранта РФФИ 15-03-04769-а.

\section{Литература/References}

1. Szymura S., Sojka L. Journal of Materials Science. 1979, № 14, p. 1890 - 1892, doi:10.1007/BF00551028

2. Kozlov Yu.I., Rakitina Z.A., Balabanova S.V. Metal Science and Heat Treatment. 1985, Vol. 27 (4), p. 293 -295, doi:10.1007/BF00652097

3. Kubota T., Wakui G., Itakagi M. IEEE Transactions on Magnetics. 1998. Vol. 34 (6). p. 3888-3896, doi:10.1109/20.728299

4. Artamonov E.V., Libman M.A., and Rudanovskii N.N. Steel in Translation. 2007, Vol. 37, №6, p. 547-551, doi:10.3103/S0967091207060186

5. Libman M.A. Materialovedenie [Material science]. 2010, №9, p. 58-64. (in Russian). [Либман M.A. Материаловедение. 2010. № 9. с. 58 - 64.]

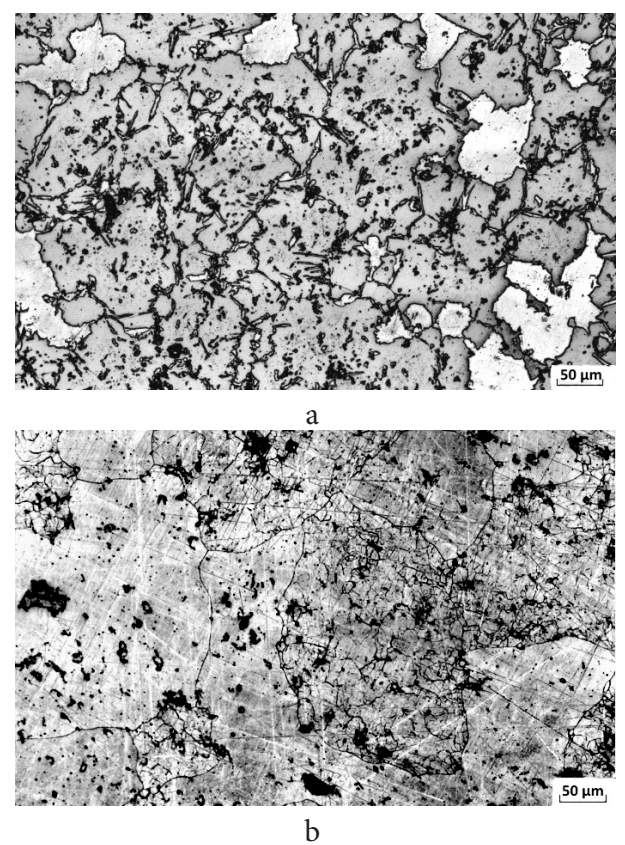

Рис. 4. Микроструктура сплава Fe-26\%Cr-16\%Co-2\%Мо-2\%W после полного цикла термообработки. Режимы спекания: $1100^{\circ} \mathrm{C}(\mathrm{a}), 1200^{\circ} \mathrm{C}(\mathrm{b})$.

Fig. 4. Electron images of $\mathrm{Fe}-26 \% \mathrm{Cr}-16 \% \mathrm{Co}-2 \% \mathrm{Mo}-2 \% \mathrm{~W}$ alloy microstructure after complete thermal treatment cycle. Sintering conditions are: (a) $1100^{\circ} \mathrm{C}$ and (b) $1200^{\circ} \mathrm{C}$.

6. Zhen Liang, Sun Xue-yin, Xu Cheng-yan, Gao Runsheng, Xu Ren-gen, Qin Lu-chang. Transactions of Non-ferrous Metals Society of China. 2007. Vol. 17. p. 346 - 350, doi:10.1016/S1003-6326 (07) 60096-X

7. Belozerov, E. V., Mushnikov N. V., Ivanova G. V. et. al. The Physics of Metals and Metallography. 2012, Vol. 113, № 4, p. 319-325, doi:10.1134/S0031918X12040023

8. Green M.L., Sherwood R.C., Wong C.C. J. Appl. Phys. 1982, Vol. 53, №.3, p. 2398 -2400, doi:10.1063/1.330824

9. Shatsov, A. A. Metal Science and Heat Treatment., 2004, vol. 46, p. 152 - 155, doi:10.1023/B:MSAT.0000036668.4 8856.02

10. Alymov M.I., Ankudinov A.B., Zelenskiy V.A., Milyaev I.M., Yusupov V.S., Ustyuhin A.S. Phizika I khimiya obrabotki materialov. [Physics and chemistry of materials treatment] 2011. № 3. p. 34 - 38. (in Russian) [Алымов М.И., Анкудинов А.Б., Зеленский В.А., Миляев И.М., Юсупов В.С., Устюхин А.С. Физика и химия обработки материалов. 2011. № 3. с. 34 - 38.]

11. Schiller S., Heisig U. Panzer S. Electron Beam Technology. New York.: Wiley \& Sons, 1982, 508 p.

12. Ustyuhin A.S., Alymov M.I., Milyaev I.M. Letters on materials. 2014. Vol. 4, №1. p. 59-61. (in Russian) [Устюхин А.С., Алымов М.И., Миляев И.М. Письма о материалах. 2014. Т. 4, № 1. с. 59 - 61.] doi:10.22226/2 410-3535-2014-1-59-61

13. Ustyuhin A.S., Alymov M.I., Krishenik P.M., Levinsky Y.V., Rogachev S.A. Phizika I khimiya obrabotki materialov [Physics and chemistry of materials treatment]. 2016, №3, p. 46-51. (in Russian) [Устюхин А.С., Алымов М.И., Кришеник П.М., Левинский Ю. В., Рогачев С. А. Физика и химия обработки материалов. 2016, № 3, с. 46 - 51.] 